\title{
High prevalence of porcine Hokovirus in German wild boar populations
}

\author{
Cornelia Adlhoch $^{1 *}$, Marco Kaiser ${ }^{1,2}$, Heinz Ellerbrok ${ }^{1}$, Georg Pauli ${ }^{1}$
}

\begin{abstract}
Porcine Hokovirus (PHoV) was recently discovered in Hong Kong. This new Parvovirus of pigs is closely related to the human Parvoviruses 4 and 5 (PARV4/5) and bovine Hokovirus (BHoV). So far, nothing is known about the presence and prevalence of PHoV in regions of the world other than Hong Kong. A study was initiated to investigate PHoV in German wild boars from five different geographical regions, using a newly established quantitative real-time PCR assay. Analysis of collected liver and serum samples revealed high overall prevalence (32.7\%; 51/156) of $\mathrm{PHoV}$ in wild boars. The prevalence differed between the regions and increased with age. Two near full-length genomes and a large fragment for three additional isolates from different regions were sequenced and used for phylogenetic analysis. The German PHoV sequences from wild boars showed a close relationship with sequences of isolates from Hong Kong.
\end{abstract}

\section{Findings}

A broad spectrum of parvoviruses is circulating worldwide in different species causing diseases in animals and humans. One of several novel animal parvoviruses is the porcine Hokovirus (PHoV), a putative member of the genus Parvovirus within the family of Parvoviridae. This new parvovirus $\mathrm{PHoV}$ has been described in pigs from Hong Kong [1]. The non-enveloped parvovirus harbours a single-stranded DNA genome of approximately $5 \mathrm{~kb}$. The genome has two open reading frames (ORFs) coding for non-structural and capsid proteins. Closely related human Parvoviruses PARV 4 and PARV5 were detected in various samples from healthy and diseased individuals [2-5].

Up to now no information is available about the presence of $\mathrm{PHoV}$ in other pig populations. This study was initiated to analyse PHoV in German wild boars. Wild boars in Germany are carrier of Hepatitis E virus (HEV) and it was of interest to analyse whether this species habours additional viruses with a zoonotic potential [6].

Liver, serum and bile samples from a total of 156 wild boars were tested for the presence of PHoV genomes. Samples $(\mathrm{n}=127)$ were collected during the hunting season 2007/2008 at several sites in Germany. Collection points in the different federal states were described in a

\footnotetext{
* Correspondence: adlhochc@rki.de

'Robert Koch-Institut, Centre for Biological Security ZBS1, Nordufer 20, 13353 Berlin, Germany
}

previous study on HEV [6]. Additional samples (9 wild boar livers) were collected at sites in the federal state of Hesse (HE) near Herleshausen/Werra, Bauhaus, Oberellen, Friedewald and Lengers between January and March 2008 and 20 wild boar serum samples were collected between November 2005 and January 2006 in the federal state of Baden Württemberg (BW) at different sites in the nature reserve Schönbuch which were nearly identical to the later sampling places in the hunting season $2007 / 2008$. In general, sampling, age determination of animals, storing and handling of samples were carried out as published previously [6]. Briefly, liver samples (20 to $40 \mathrm{mg}$ ) were homogenized in $500 \mu \mathrm{l}$ PBS using Precellys ceramic beads (diameter of $1.4 \mathrm{~mm}$; Peqlab Biotechnology, Erlangen, Germany) and the FastPrep ${ }^{\oplus}$ FP220A homogenizer (Qbiogene, MP Biomedicals, Solon, OH, USA). A volume of $200 \mu$ of supernatant of the centrifugated homogenized liver, bile or serum samples was used for DNA extraction using the NucleoS$\operatorname{pin}^{\oplus}$ Blood preparation kit (Macherey-Nagel, Düren, Germany). A quantitative real-time PCR (qPCR) assay using the PHoV_TM 5' nuclease probe (TaqMan ${ }^{\circledR}$ probe) in combination with 3 primers $P H o V_{-}$F/PHoV_R/HPV_R (Table 1; TIB MOLBIOL, Berlin, Germany) was applied in this study to determine the copy numbers of $\mathrm{PHoV}$ genomes. The assay was established to detect the newly described parvovirus PHoV and the human PARV4/ PARV5 using primers binding within a conserved region 
Table 1 Overview of the analysed samples for PHoV

\begin{tabular}{lccccc}
\hline Region & $\mathbf{n}_{\text {positive }} / \mathbf{n}_{\text {total }}$ & $<1$ year & $\mathbf{1 - 2}$ years & Adult & Unknown \\
\hline BW_2005 & $\begin{array}{c}2 / 20 \\
(10.0 \%)\end{array}$ & $0 / 9$ & $2 / 8$ & $0 / 3$ & $0 / 0$ \\
BW_2007 & $\begin{array}{c}5 / 27 \\
(18.5 \%)\end{array}$ & $0 / 10$ & $2 / 7$ & $0 / 4$ & $3 / 6$ \\
HE & $2 / 9$ & $1 / 3$ & $1 / 4$ & $0 / 2$ & $0 / 0$ \\
RP & $(22.2 \%)$ & & & & \\
& $4 / 53$ & $1 / 27$ & $0 / 14$ & $3 / 11$ & $0 / 1$ \\
BB & $(7.5 \%)$ & & & & \\
SA & $12 / 19$ & $4 / 9$ & $3 / 3$ & $3 / 3$ & $2 / 4$ \\
& $(63.2 \%)$ & & & & \\
\hline Total & $26 / 28$ & $9 / 9$ & $10 / 10$ & $7 / 9$ & $0 / 0$ \\
n C & $(92.9 \%)$ & & & & \\
\%/Pos & $51 / 156$ & $15 / 67$ & $18 / 46$ & $13 / 32$ & $5 / 11$ \\
\%/Total & $(32.7 \%)$ & $(22.4 \%)$ & $(39.1 \%)$ & $(40.6 \%)$ & $(45.5 \%)$ \\
\hline & 17 & 7 & 9 & 1 & 0 \\
& 33.3 & 46.7 & 50.0 & 7.7 & 0 \\
& 10.9 & 10.5 & 19.6 & 3.1 & 0 \\
\hline
\end{tabular}

Number of PHoV positive and total tested animals with prevalences and virus load ( $\mathrm{C}_{\mathrm{T}}$-values) overall and divided by age groups and regions of sample collection.

*: estimated percentages per total samples tested positive; ${ }^{\dagger}$ : estimated percentages per total samples tested; n: number of animals; BW: Baden Württemberg, HE: Hesse, RP: Rhineland Palatinate, BB: Brandenburg, SA: Saxony

for each virus. DNA samples in a volume of $2.5 \mu$ l were analysed using the following qPCR protocol in a final volume of $25 \mu \mathrm{l}$ with $10 x b u f f e r, 4 \mathrm{mM}$ of $\mathrm{MgCl}_{2}$, dNTP $0.2 \mathrm{mM}$ each, $0.3 \mu \mathrm{M}$ of each primer, $0.1 \mu \mathrm{M}$ of probe, ROX $0.1 \mu \mathrm{M}$ and Platinum ${ }^{\circledR}$ Taq $0.5 \mathrm{U}^{\text {U Platinum }}{ }^{\circledR}$ Taq DNA polymerase, $\mathrm{MgCl}_{2}$ and dNTPs were obtained from Invitrogen (Carlsbad, CA, USA) and water (Molecular Biology Grade) from Eppendorf (Hamburg, Germany). General reaction conditions for the real-time assay were $95^{\circ} \mathrm{C}$ for $10 \mathrm{~min}$ and 45 cycles with $95^{\circ} \mathrm{C}$ for $15 \mathrm{sec}, 60^{\circ} \mathrm{C}$ for 35 sec. Reactions were run in an ABI GeneAmp ${ }^{\circledR}$ 7500 Detection System (Applied Biosystems, Foster City, CA, USA). Plasmid pHoko containing the 83 nucleotide (nt) amplification product from the isolate PHoV_BW2117 [GQ869539] was established. Insert was verified by sequencing and copy numbers in this preparation were calculated using standard methods. The plasmid was tenfold serially diluted in water containing $\gamma$ DNA $(1 \mathrm{ng} / \mu \mathrm{l})$ from $10^{6}$ copies to 1 copy as standards for quantification of viral genomes. For qPCR each sample was analysed in duplicate. Copy numbers in samples were determined using a standard curve. The detection limit was estimated to be 10 copies of DNA per reaction. The $\beta$-Actin-qPCR assay was used as internal control [6]. The near full-length genomes were generated with PCR and nested PCR using several primer pairs in combination with primers for sequencing (Table 1) with the Platinum $^{\circledast}$ Taq DNA polymerase kit as described previously for HEV [6]. Sequence of amplicons was determined either directly using the PCR product or after cloning into vector $\mathrm{pCR}$ II TOPO (Invitrogen) by sequencing both strands with the Big Dye3.1 protocol using an automated sequencer (Genetic Analyzer 3130 xl, Applied Biosystems). Sequence data were analysed using ABI PRISM DNA Sequencing Analysis Software (Version 3.7, Applied Biosystems). Phylogenetic tree analysis was performed using MEGA 4.01 [7] program http://www.megasoftware. net and BLAST network program (National Center for Biotechnology Information, Bethesda, MD, USA).

The prevalence of $\mathrm{PHoV}$ in liver or serum samples of wild boars differed between sampling regions: While a low prevalence was seen in Rhineland Palatinate (RP), BW and HE, the samples collected in Saxony (SA) and Brandenburg (BB) showed high values (Table 2). The overall prevalence was $32.7 \%$ (51/156), 17 of 51 (33.3\%) animals tested positive with $\mathrm{C}_{\mathrm{T}}$-values lower than 30 indicating high copy numbers of more than $10^{6}$ genome equivalents per $\mathrm{mg}$ of liver tissue, 16 of the 17 animals (94\%) showing high copy numbers were below 2 years of age ( 7 animals $<1$ year, 9 animals $1-2$ years). The analysis of the age distribution showed an increase in prevalence for animals older than 1 year, but the highest proportion of animals with high virus loads was seen in the group below 2 years of age (Table 1). Corresponding serum and liver tissue samples from six animals were tested in parallel showing comparable values for both compartments. Although it was shown previously that HEV was detected in high virus load in bile samples [6], quantification for $\mathrm{PHoV}$ in samples from three animals yielded virus loads that were up to 1000 times lower in bile samples than in the liver (data not shown). This result implicates, that $\mathrm{PHoV}$ has an organ tropism different from HEV. It can be assumed that a high virus

Table 2 Used primers and probe for the quantitative analysis and generation of genome fragments of PHoV

\begin{tabular}{ll}
\hline Primer name & Orientation 5'-3' $^{\prime}$ \\
\hline PHoV_F & gTT ggT CCT ggT AAT CCT YTg g \\
PHoV_R & TCg TAC CgT TCA TCg Tgg Tg \\
HPV_R & TgC gTA CCg TTC ATC ATg ATg TT \\
PHoV_TM & FAM-Agg gAC CAg Tgg ATg ARg CAg C-BBQ \\
PHoV_240F & CAC ACC TAC CTC gCC TAT AAg AAT C \\
PHoV_1273F & ggT AYT TTg CWg CHT ggg C \\
PHoV_1408R & CAA TTC ACR CAR CCR TAA gAW ggA \\
PHoV_1847F & CCg ATC TCC CCg TCT gCC \\
PHoV_2293F & CCg CAC TgA ggg CTA Cg \\
PHoV_2492F & ggT AAg MAA WCA TgW CWg CYg C \\
PHoV_2492R & gCR gCW gWC ATg WTT KCT TAC C \\
PHoV_4115F & ggg ARA ATT ATg TTY TKC CTC ART ATg g \\
PHoV_4395R & ATC WAC MCC TgT CAT RAT MgC \\
PHoV_5288R & CAC TgA TCA gAA ggM ACY TCR TAC AC \\
\hline
\end{tabular}

F: forward; R: reverse orientation; TM: TaqMan probe 
load of PHoV in liver tissue and serum indicates an acute or persistent infection with a simultaneous viraemia.

In order to analyse the phylogenetic relationship between PHoV in Hong Kong and in Germany near full-length genome sequences with $4942 \mathrm{nt}$ and $4944 \mathrm{nt}$ were amplified from isolates BW2117 [GQ869539] and Sa15 [GQ869540], respectively. Additionally, discontinuous genome sequences of isolates from isolate BW22 [GQ869543], RP1754 [GQ869541] and BB09 [GQ869542] were generated with total sizes of 4564, 3027 and 3928 nt, respectively. All isolates were incomplete at the 3'-end of the VP1 and VP2 ORFs. The phylogenetic analysis showed that the $\mathrm{PHoV}$ isolates from German wild boars were closely related to Hong Kong isolates but formed a separate branch in the phylogenetic tree of all known porcine, bovine and human isolates from the GeneBank database (Figure 1). All German sequences were closely related to each other. The generated full-length sequences BW2117 and Sa15 differed in 44 of 4796 nucleotides (99.1\% identity). A divergence of up to $40 \%$ was found to complete PARV4/ 5 sequences, and of $37 \%$ to bovine isolates. Compared to the isolates from Hong Kong a difference of 1.8-2.0\% (Sa15) and 2.0-2.3\% (BW2117) was seen for the German full-length sequences on nucleotide level.

Within NS1 7 (1.1\%) unique amino acid exchanges were observed in the German isolates BW2117 and Sa15 in comparison to the Hong Kong isolates The

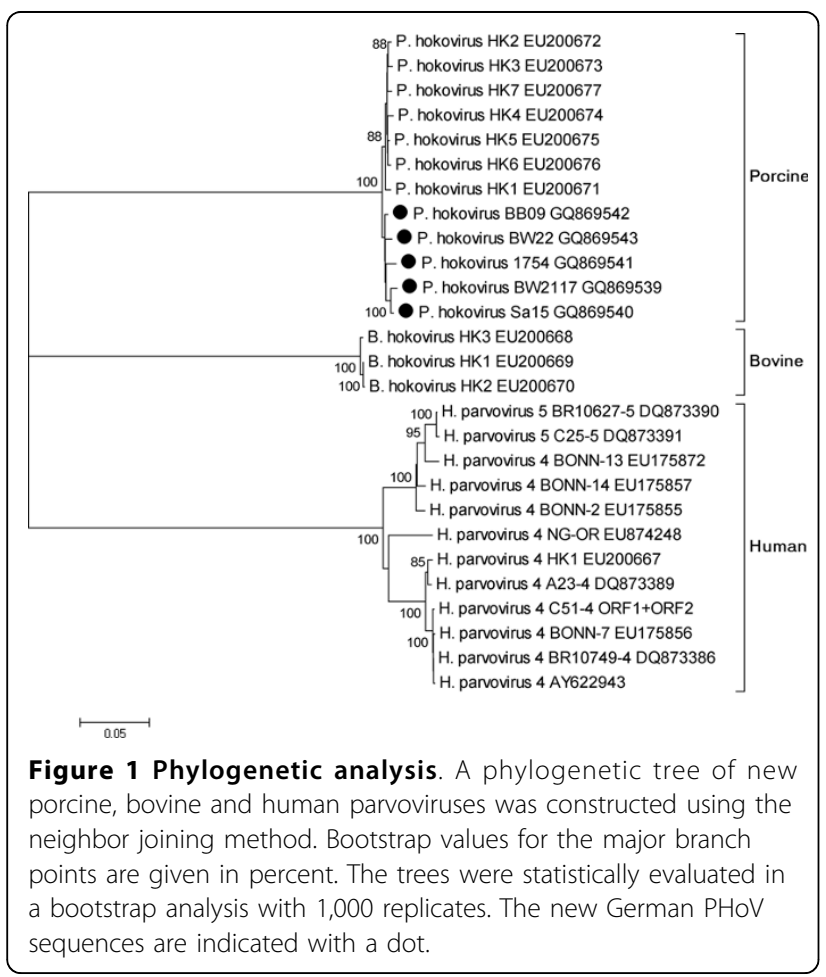

phylogenetic analysis showed that within the ORFs coding for VP1 and VP2 proteins only one (VP1; 0.2\%) and three (VP2; $0.3 \%$ ) unique amino acid exchanges were found in the German isolates (BW2117 and Sa15) in comparison to the Hong Kong isolates.

In this study it was shown that the newly discovered $\mathrm{PHoV}$ is present in European wild boar populations. The virus was detectable in approximately every third animal tested. PHoV prevalence showed regional variation as determined in samples from animals collected in 5 geographic regions in Germany. The presence of high copy numbers of viral genomes in younger animals ( $\leq$ two years) points to an infection early in life. The increase of the prevalence in older animals supports the hypothesis of $\mathrm{PHoV}$ persistence in liver comparable to the situation observed for PARV4 and PARV5 infections in humans [8]. Therefore persistence might be a common feature for this new group of parvoviruses. So far, no clear disease has been linked to the infection or persistence of these new parvoviruses. The phylogenetic analysis showed a close relationship of the German PHoV sequences with the isolates from Hong Kong, although the European isolates clustered together in one separate branch. It can be speculated that the virus has been distributed through pigs that have been imported from Europe to Hong Kong.

Although the qPCR assay was established to detect all known isolates of the new Parvovirus group (PARV4, PARV5, PHoV and BHoV), only $\mathrm{PHoV}$ was found in the wild boar samples. The fact that approximately 600.000 wild boars are shot and consumed every year in Germany clearly highlights a potential route for a zoonotic transmission to humans. While the prevalence of $\mathrm{PHoV}$ in commercial pigs is yet unknown $\mathrm{PHoV}$ has been detected in a variety of porcine tissues with high virus load [1] indicating yet another potential risk of zoonotic transmission of PHoV to humans that urgently needs to be evaluated.

Acc. numbers of generated sequences:

[P._Hokovirus_BW2117: GQ869539; P._Hokovirus_Sa15: GQ869540; P._Hokovirus_1754: GQ869541; P. _Hokovirus_BB09: GQ869542; P._Hokovirus_BW22: GQ869543]

\section{Abbreviations}

BB: Brandenburg; SA: Saxony; RP: Rhineland Palatinate; BW: Baden Württemberg; HE: Hesse; qPCR: quantitative real-time PCR; PHoV: porcine Hokovirus; BHoV: Bovine Hokovirus; HEV: Hepatitis E virus

\section{Acknowledgements}

The authors thank Anna Löwa and Markus Ulrich for excellent technical support and Ursula Erikli for copy-editing. The authors are grateful to $P$. Linderoth at the LWG Aulendorf (BW), who provided samples from the nature reserve, U. Hohmann and D. Huckschlag from the FAWF Trippstadt (RP), H.-J. Hormel at the forestry management (BW) and the forest officials J. P. Huber in Welzow-Proschim (SA), F. Wehnert in Krausnick (BB) and D. 
Preißel-Baranowsky (HE) as well as all collaborating hunters for the opportunity and the support in collecting wild boar samples.

\section{Author details}

'Robert Koch-Institut, Centre for Biological Security ZBS1, Nordufer 20, 13353

Berlin, Germany. ${ }^{2}$ GenExpress GmbH, Eresburgstr. 22-23, 12103 Berlin,

Germany.

\section{Authors' contributions}

CA: Study design, sampling, interpretation of the data and manuscript draft. MK: Sample analysis, phylogenetic analysis, interpretation of the data and manuscript draft. HE: Critical interpretation of the data and manuscript draft. GP: Study design, interpretation of the data and approval of the manuscript. All authors have read and approved the final manuscript.

\section{Competing interests}

The authors declare that they have no competing interests.

Received: 21 May 2010 Accepted: 25 July 2010 Published: 25 July 2010

\section{References}

1. Lau SK, Woo PC, Tse H, Fu CT, Au WK, Chen XC, Tsoi HW, Tsang TH, Chan JS, Tsang DN, et al: Identification of novel porcine and bovine parvoviruses closely related to human parvovirus 4. J Gen Virol 2008, 89:1840-1848.

2. Fryer JF, Kapoor A, Minor PD, Delwart E, Baylis SA: Novel parvovirus and related variant in human plasma. Emerg Infect Dis 2006, 12:151-154.

3. Fryer JF, Delwart E, Hecht FM, Bernardin F, Jones MS, Shah N, Baylis SA: Frequent detection of the parvoviruses, PARV4 and PARV5, in plasma from blood donors and symptomatic individuals. Transfusion (Paris) 2007, 47:1054-1061.

4. Jones MS, Kapoor A, Lukashov W, Simmonds P, Hecht F, Delwart E: New DNA viruses identified in patients with acute viral infection syndrome. J Virol 2005, 79:8230-8236.

5. Tuke PW, Parry RP, Appleton H: Parvovirus PARV4 visualisation and detection. J Gen Virol 2010, 91(Pt2):541-544.

6. Adlhoch C, Wolf A, Meisel H, Kaiser M, Ellerbrok H, Pauli G: High HEV presence in four different wild boar populations in East and West Germany. Vet Microbiol 2009, 139:270-278.

7. Tamura K, Dudley J, Nei M, Kumar S: MEGA4: Molecular Evolutionary Genetics Analysis (MEGA) software version 4.0. Mol Biol Evol 2007, 24:1596-1599.

8. Schneider B, Fryer JF, Reber U, Fischer HP, Tolba RH, Baylis SA, EisHubinger AM: Persistence of novel human parvovirus PARV4 in liver tissue of adults. J Med Virol 2008, 80:345-351.

\section{Submit your next manuscript to BioMed Central} and take full advantage of:

- Convenient online submission

- Thorough peer review

- No space constraints or color figure charges

- Immediate publication on acceptance

- Inclusion in PubMed, CAS, Scopus and Google Scholar

- Research which is freely available for redistribution

Submit your manuscript at www.biomedcentral.com/submit 